Boletín de la Sociedad Geológica Mexicana

VOLUMEN 63, NÚM. 3, 2011, P. 479-486

\title{
Enmiendas orgánicas y durazno, Prunus persica (L.) Batsch, en el mejoramiento de una capa endurecida, tepetate tipo duripán
}

\author{
David Flores-Román ${ }^{1, *}$, Carla Guadalupe Muñiz-Irigoyen ${ }^{1}$, \\ María del Socorro Galicia-Palacios ${ }^{2}$, Ronald Ferrera-Cerrato ${ }^{3}$, Gerardo Zenteno ${ }^{4}$ \\ ${ }^{1}$ Departamento de Edafología, Instituto de Geología, Universidad Nacional Autónoma de México, Ciudad Universitaria, 04510 , \\ México, D.F. \\ ${ }^{2}$ Facultad de Ciencias, Universidad Nacional Autónoma de México, Ciudad Universitaria, 04510, México, D.F. \\ ${ }^{3}$ Instituto de Recursos Naturales, Colegio de Posgraduados, Carretera México-Texcoco km 36.5, Montecillo, Texcoco, 56230, Estado \\ de México. \\ ${ }^{4}$ Instituto de Geología, Universidad Nacional Autónoma de México, Ciudad Universitaria, 04510, México, D.F. \\ *davidf@servidor.unam.mx
}

\section{Resumen}

Con el fin de mejorar la calidad productiva de los tepetates y con ello su habilitación, el objetivo de este estudio fue evaluar el efecto del durazno (Prunus persica (L)Batsch), abonos orgánicos y micorriza en las características físicas, químicas y bioquímicas de un tepetate fragmentado. Este trabajo se realizó en invernadero en el Instituto de Geología, Universidad Nacional Autónoma de México (UNAM). El tepetate se recolectó en el municipio de Tetela del Volcán, Morelos, se roturó (2 a 10 mm diámetro) y se colocó en macetas sin perforaciones, adicionándose las enmiendas: estiércol bovino, composta y micorriza. Las plantas de durazno se trasplantaron de tres meses de edad; al final del experimento (un año) se cortaron, se sacó el tepetate de la maceta y se secó al aire para su análisis en el laboratorio. El diseño experimental fue completamente al azar con dos factores y tres niveles cada uno ( 9 tratamientos), con cuatro réplicas (36 unidades experimentales). Con los datos se hizo un análisis de varianza y se usó la prueba de Tukey ( $\mathrm{p} \leq 0.05$ ) en las medias. Los valores de agregación presentaron el mayor porcentaje en la fracción de 5 a $10 \mathrm{~mm}$ y la de menor porcentaje fue la $>10$ $\mathrm{mm}$; sin embargo, en las distribuciones de los tratamientos relativas a la distribución original del material utilizado, se observa una modificación importante, comparada con la experimentada en el testigo absoluto. En la estabilidad, la fracción con mayor porcentaje fue la $>5 \mathrm{~mm}$ y la de menor de 3 a $5 \mathrm{~mm}$. El pH fue de ligera a medianamente alcalino, el carbono orgánico aumentó. De las bases intercambiables el sodio $\left(\mathrm{Na}^{+}\right)$obtuvo incremento significativo. La humificación es incipiente por el predominio de estructuras alifáticas y poco tiempo para que evolucionen las moléculas. Los polisacáridos se incrementaron en los tratamientos donde no se aplicó inóculo.

Palabras clave: capas duras, habilitación, Prunus persica.

\begin{abstract}
The objective of this study was to evaluate the effect of peach, Prunus persica $(L)$ Batsch, organic emendations and mycorrhizae on the physical, chemical and biochemical characteristics of a fragmented tepetate with the intention of rehabilitating it and improving its productive quality. This work was carried out in a greenhouse at the Instituto de Geología, Universidad Nacional Autónoma de México. Tepetate was collected in the Tetela del Volcán municipality, State of Morelos, Mexico, broken up into fragments (2 to 10 mm in diameter) and arranged in pots without holes, adding emendations: cow manure, compost and mycorrhiza. The peach plants were trasplanted at an age of three months and were cut at the end of the experiment (one year). The tepetate was taken out of the pot and air dried for laboratory analysis. Experimental design was completely random with two factors and three levels each (9 treatments), with four repetitions (36 experimental units). A variance analysis was performed with the data and the Tukey proof was used in means
\end{abstract}


$(p \leq 0.05)$. The aggregation values presented the biggest percentage in fraction 5-10 $\mathrm{mm}$ and the lowest percentage was fraction $>$ $10 \mathrm{~mm}$; however, the relative distribution compared to the original material distribution shows an important modification compared to the changes in the absolute witness. In the stability test, the fraction with the biggest percentage was $>5 \mathrm{~mm}$ and the lowest, 3-5 $\mathrm{mm}$. The $\mathrm{pH}$ varied from light to medium alkalinity, and organic carbon increased. $\mathrm{Na}^{+}$increased significantly. The process of humus formation is incipient because of the predominance of aliphatic structures and insufficient time for molecular evolution. Polysaccharides increased in treatments that were not inoculated.

Keywords: hard layers, rehabilitation, Prunus persica.

\section{Introducción}

El término tepetate incluye muchos materiales que tienen en común niveles de cementación que pueden ser desde muy débiles hasta extremadamente fuertes, o grados de compactación que varían de medios a fuertes (Flores et al., 1991). Los tepetates son materiales de origen volcánico con aportes secundarios de cementantes de origen edafológico (Zebrowski, 1992). Bajo condiciones naturales, los tepetates tienen porosidad total y capacidad de aireación bajas, infiltración lenta y la vegetación herbácea que soportan es escasa (Báez-Pérez et al., 2007). El establecimiento de especies cultivadas es prácticamente imposible, debido a su dureza, muy bajo contenido de materia orgánica, de nitrógeno $(\mathrm{N})$ y fósforo $(\mathrm{P})$, baja capacidad de almacenamiento de agua, estructura masiva y densidad aparente alta. Su manejo requiere prácticas y equipo cuyo costo resulta inaccesible para los productores, por lo que se les abandona y se les considera áreas improductivas (Velázquez et al., 2001). Tales capas endurecidas pueden habilitarse, principalmente a un uso frutícola o pecuario si se induce el proceso de fragmentación-agregación, la adición de materia orgánica y el establecimiento de plantas. El presente trabajo es parte de una línea de investigación tendiente a la habilitación de estos materiales, donde se han probado diferentes enmiendas, tanto orgánicas como inorgánicas, así como, diversas especies vegetales cultivadas de ciclo corto, y perennes. El objetivo de este estudio fue evaluar el efecto del durazno Prunus persica (L.) Batsch, abonos orgánicos y micorriza en las características físicas, químicas y bioquímicas de un tepetate fragmentado, para mejorar su calidad productiva y su habilitación.

\section{Materiales y métodos}

Este trabajo se realizó en invernadero en el Instituto de Geología, UNAM. El material se recolectó en el municipio de Tetela del Volcán, Estado de Morelos, México (18 $57^{\circ}$ $48^{\prime}$ " N y 99 15' 12" O, a 2040 msnm). El tepetate es de tipo duripán (Velázquez et al., 2001), de color pardo amarillento claro en seco (10YR 6/4) y pardo amarillento oscuro en húmedo (10YR 3/4) y su clasificación textural es franca. El $\mathrm{pH}$ es neutro (7.1), la materia orgánica es muy baja $(0.2$
$\%$ ), la capacidad de intercambio catiónico es media-alta (16.7 $\left.\mathrm{cmol}^{+} \mathrm{kg}^{-1}\right)$, las bases intercambiables son altas $\left(\mathrm{Ca}^{++}\right.$ $10 \mathrm{cmol}^{+} \mathrm{kg}^{-1}, \mathrm{Mg}^{++} 6.2 \mathrm{cmol}^{+} \mathrm{kg}^{-1}, \mathrm{Na}^{+} 1.3 \mathrm{cmol}^{+} \mathrm{kg}^{-1} \mathrm{y} \mathrm{K}^{+}$ $1.2 \mathrm{cmol}^{+} \mathrm{kg}^{-1}$ ) y el porcentaje de saturación de bases es alto $(100 \%)$. Se utilizó el durazno por ser un frutal altamente cultivado en el municipio antes citado, al noreste del estado de Morelos, donde existen grandes áreas erosionadas con este material endurecido.

El tepetate se roturó manualmente en fragmentos (2 a 10 mm diámetro) con la siguiente distribución de porcentajes: de 10 a $5 \mathrm{~mm}, 49.73 \%$; de 5 a $2 \mathrm{~mm}, 39.65 \%$; de 2 a $1 \mathrm{~mm}, 4 \%$; de 1 a $0.25 \mathrm{~mm}, 4 \%$ y $<0.25,2.62 \%$. Se colocó en macetas de plástico con capacidad de $5 \mathrm{~kg}$ sin perforaciones para evitar pérdidas, adicionándose en seco las enmiendas estiércol bovino ( $0.94 \%, \mathrm{C} 7.25 \%, \mathrm{~K}$ $11.13 \mathrm{cmol}^{+} \mathrm{kg}^{-1}$ ) $180 \mathrm{~g} \mathrm{maceta}^{-1}$ (equivalente a $72 \mathrm{Mg} \mathrm{ha}^{-1}$ ) y composta (N $1.79 \%$, C $18.11 \%$, K $20 \mathrm{cmol}^{+} \mathrm{kg}^{-1}$ ) $100 \mathrm{~g}$ maceta $^{-1}$ (equivalente a $40 \mathrm{Mg} \mathrm{ha}^{-1}$ ) y rotulándose según los tratamientos. El material resultante se considera un Antrosol hórtico (IUSS-WRB, 2006). Las plantas se establecieron de tres meses de edad a raíz desnuda; antes del transplante se aplicó a las raíces $20 \mathrm{~g}$ de inóculo de Glomus spp. por planta. Al final del experimento (un año) se tomaron muestras del tepetate de cada tratamiento para su análisis en laboratorio. En el invernadero la temperatura osciló entre 18 y $24^{\circ} \mathrm{C}$, la humedad relativa fue $60 \%$ y hubo una luminosidad de 11 $\mathrm{h}$ en invierno y $13 \mathrm{~h}$ en verano.

Con base en lo anterior se establecieron los tratamientos siguientes (Tabla 1): el tratamiento testigo absoluto (TAB) consistió solamente en el tepetate fragmentado en su maceta al cual solo se le agregó agua, al mismo tiempo que a los otros tratamientos. El testigo absoluto cero (TABo) fue el tepetate tal como se trajo del campo. El testigo con estiércol (TE) fue igual que TAB pero se la agregó estiércol. El testigo con composta (TC) fue igual que TAB, pero con adición de composta. El tratamiento planta $(\mathrm{P})$ consistió en la planta de durazno con el tepetate fragmentado. Planta estiércol (PE) fue tepetate fragmentado, durazno y estiércol. Planta composta (PC) fue tepetate fragmentado, durazno y composta. Planta inóculo (PI) fue tepetate fragmentado, durazno más inóculo. Planta inóculo estiércol (PIE) tepetate fragmentado, durazno, inóculo y estiércol. Planta inóculo composta (PIC) tepetate fragmentado, durazno, inóculo y composta. 
Tabla 1. Tratamientos establecidos.

\begin{tabular}{cccc}
\hline Tratamiento & Planta & Enmienda & Clave \\
\hline 1 & Sin & Sin & TAB \\
2 & Sin & Estiércol & TE \\
3 & Sin & Composta & TC \\
4 & Con & Sin & P \\
5 & Con & Estiércol & PE \\
6 & Con & Composta & PC \\
7 & Con +Inóculo & Sin & PI \\
8 & Con + Inóculo & Estiércol & PIE \\
9 & Con + Inóculo & Composta & PIC \\
\hline P
\end{tabular}

TAB=testigo; $\mathrm{E}=$ estiércol; $\mathrm{C}=$ composta; $\mathrm{P}=$ planta; $\mathrm{I}=$ inóculo

Después del experimento, las plantas se cortaron al ras, se sacó todo el material de la maceta y se secó al aire para dejar los agregados como se obtuvieron. Mediante cuarteos (muestra representativa), se tomaron $1.5 \mathrm{~kg}$ para agregación y $100 \mathrm{~g}$ para estabilidad. Las pruebas físicas, agregación y estabilidad, se hicieron según Kemper y Rosenau (1986). Los análisis químicos fueron: 1) pH, se determinó en agua $\left(\mathrm{H}_{2} \mathrm{O}\right)$-suelo, relación 1:2.5 con un potenciómetro Orión modelo 920A; 2) materia orgánica por el método de oxidación vía húmeda con dicromato de potasio $\left(\mathrm{K}_{2} \mathrm{Cr}_{2} \mathrm{O}_{7}\right)$ en medio ácido (Nelson y Sommers, 1996); 3) Capacidad de intercambio catiónico según Jackson (1970), usando cloruro de calcio $\left(\mathrm{CaCl}_{2}\right) 1 \mathrm{~N} \mathrm{pH} 7$ para saturar la muestra, se lavó con alcohol etílico y saturó con cloruro de sodio $(\mathrm{NaCl})$ $1 \mathrm{~N} \mathrm{pH} \mathrm{7;4)} \mathrm{las} \mathrm{bases} \mathrm{intercambiables} \mathrm{se} \mathrm{extrajeron} \mathrm{por} \mathrm{el}$ método del acetato de amonio $1 \mathrm{~N} \mathrm{pH} \mathrm{7;} \mathrm{5)} \mathrm{el} \mathrm{Ca} \mathrm{y} \mathrm{el} \mathrm{Mg}$ se titularon usando versenato; 6) el $\mathrm{K}$ y $\mathrm{Na}$ intercambiables se determinaron en un flamómetro Corning 400. Los análisis bioquímicos fueron: 1) el carbono orgánico se midió por el método de oxidación con dicromato de potasio (Nelson y Sommers, 1996); 2) los polisacáridos totales por la técnica del ácido-fenol-sulfúrico (Ivarson y Sowden, 1962). La fracción húmica total se extrajo con una solución de hidróxido de sodio $(\mathrm{NaOH})$ y pirofosfato de sodio $\left(\mathrm{Na}_{4} \mathrm{P}_{2} \mathrm{O}_{7}\right)$ $1 \mathrm{M}$ (Kononova, 1982); los ácidos húmicos (AH) se separaron de los fúlvicos (AF) por precipitación con ácido sulfúrico 1:1 hasta $\mathrm{pH}$ 1.5. Las dos fracciones se evaluaron con espectroscopía visible-UV; para esas determinaciones se usaron $200 \mathrm{~g}$ de tepetate, molido y tamizado a 2.0 y 0.25 $\mathrm{mm}$. La colonización de las micorrizas se determinó por tinción, con base en Ferrera et al. (1993).

El diseño experimental se hizo con dos factores (planta y enmienda) y tres niveles, cada uno fue completamente al azar; así hubo nueve tratamientos (Tabla 1), con cuatro réplicas cada uno, 36 unidades experimentales. Los efectos de tratamientos se determinaron con un análisis de varianza y la prueba de Tukey para comparar medias, para lo cual se aplicó el paquete estadístico JMP versión 5 (JMP, 2002).

Además, se analizaron las distribuciones de los agregados en cada tratamiento respecto de la distribución original. Primero se obtiene una gráfica del comportamiento de las distribuciones (Figura 1), observándose un comportamiento similar para testigo y tratamientos; posteriormente se resta el porcentaje original de agregados (TABo) a dichos tratamientos, obtenida al año, incluyendo el testigo TAB y se grafica la distribución relativa correspondiente a cada serie (Figura 2).

\section{Resultados y discusión}

\subsection{Agregación en seco}

La fracción de 10 - $5 \mathrm{~mm}$ presenta el mayor porcentaje absoluto de agregación (Tabla 2) pero esto se debe a que sigue el mismo comportamiento de la distribución del material original, en términos relativos el intervalo de $2-10$ mm presenta un decaimiento, indicando una disgregación; en cambio el intervalo de 0.25 a $2 \mathrm{~mm}$ muestra un aumento relativo a la distribución original (Figura 2), lo que indica una agregación del material disgregado en el intervalo anterior. La diferencia TAb-TAbo es la referencia obligada, los comportamientos de los tratamientos se aprecian mejor ya que se elimina el efecto inherente a las condiciones ambientales comunes. En la fracción $<0.25 \mathrm{~mm}$ hay diferencias significativas $(\mathrm{p} \leq 0.05)$ entre los tratamientos testigo+estiércol (TE) y testigo+composta (TC), pero no con los tratamientos restantes. En otro trabajo relacionado, el intervalo de agregación que predominó fue de 7.0 a 10 $\mathrm{mm}$, considerado positivo, ya que proporcionan propiedades físicas favorables para el crecimiento de los cultivos (Acevedo et al., 2001).

Al final del experimento se observó la presencia de fracciones $>10 \mathrm{~mm}$ (Tabla 2) que no estaban presentes al inicio. Estas fracciones resultaron de la disgregación y posterior agregación del tepetate. Velázquez et al. (2001) encontraron que en el tepetate fragmentado puede haber procesos de agregación y disgregación, proponiendo que los factores que incidieron en estos procesos fueron, principalmente, los ciclos de humedecimiento y secado (Shiel et al., 1988).

\subsection{Estabilidad de agregados en húmedo}

La fracción $>5 \mathrm{~mm}$ presentó el mayor porcentaje de estabilidad de agregados y el menor porcentaje de agregados de 3 a 5 mm (Tabla 3). García et al. (2009), también reportaron el mayor dominio de agregados en húmedo en la fracción $>$ $5 \mathrm{~mm}$.

Los principales factores de unión en el tepetate son la consolidación y cementación (Zebrowski, 1992; Hidalgo et al., 1997; Acevedo y Flores, 2000), por lo cual su estabilidad es grande cuando se trata de fragmentos. Sin embargo, también se presenta la unión de partículas por efecto de agregantes, como la materia orgánica, constituyendo verdaderos agregados (Denef et al., 2001). En este trabajo ambos se consideraron agregados. 
Agregación en tepetate

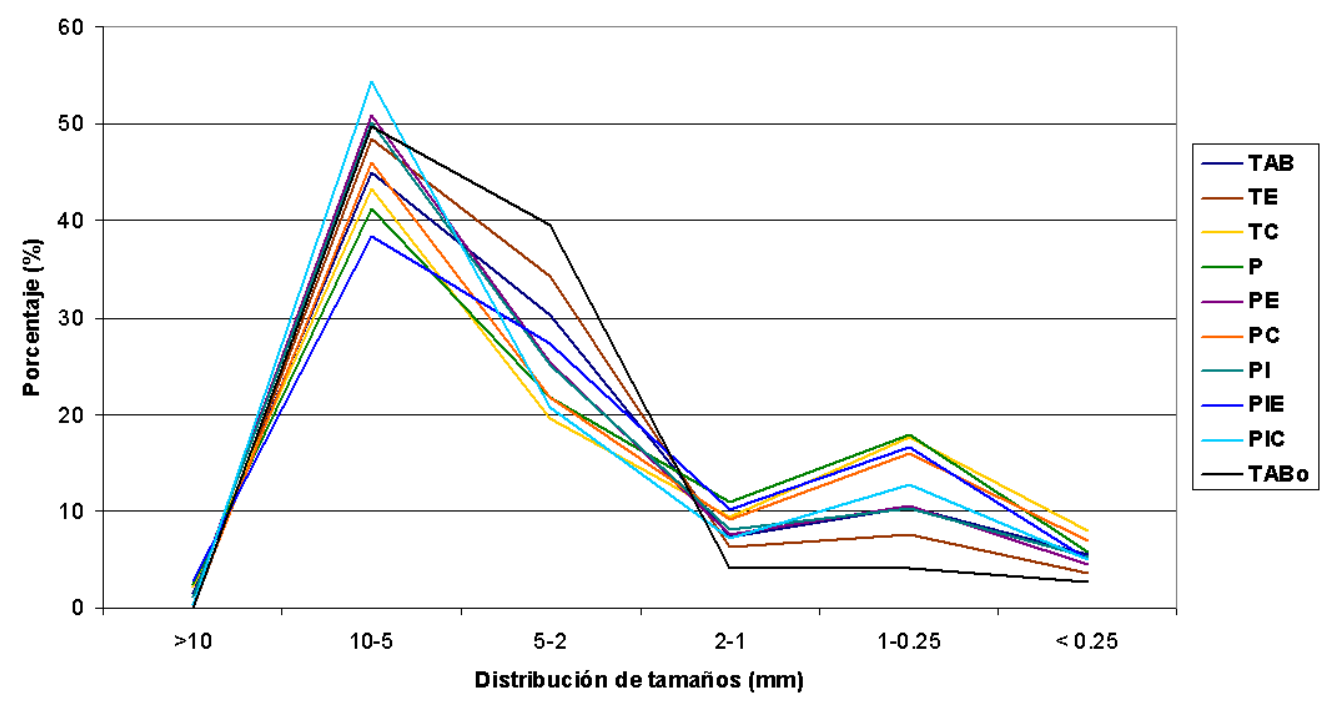

Figura 1. Distribución porcentual de tamaños de agregados en tepetate

\section{Agregación relativa de tepetate}

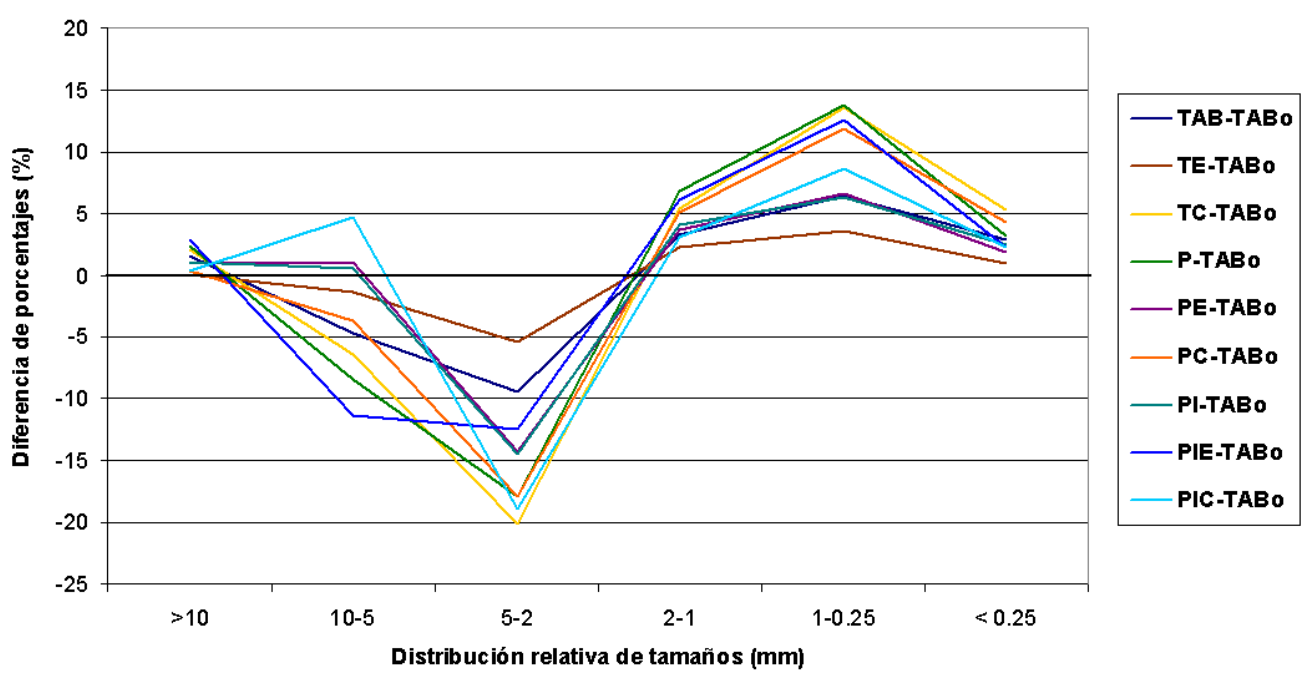

Figura 2. Distribución relativa porcentual de tamaños de agregados en tepetate.

Tabla 2. Porcentaje promedio del tamaño de agregados (mm)

\begin{tabular}{ccccccc}
\hline Tratamiento & $>10$ & $10-5$ & $5-2$ & $2-1$ & $1-0.25$ & $<0.25$ \\
\hline TAB & $1.56 \mathrm{a}$ & $45.00 \mathrm{a}$ & $30.22 \mathrm{a}$ & $7.35 \mathrm{a}$ & $10.43 \mathrm{a}$ & $5.44 \mathrm{ab}$ \\
$\mathrm{TE}$ & $0.01 \mathrm{a}$ & $48.35 \mathrm{a}$ & $34.19 \mathrm{a}$ & $6.29 \mathrm{a}$ & $7.57 \mathrm{a}$ & $3.59 \mathrm{~b}$ \\
$\mathrm{TC}$ & $2.10 \mathrm{a}$ & $43.39 \mathrm{a}$ & $19.50 \mathrm{a}$ & $9.42 \mathrm{a}$ & $17.64 \mathrm{a}$ & $7.95 \mathrm{a}$ \\
$\mathrm{P}$ & $2.45 \mathrm{a}$ & $41.26 \mathrm{a}$ & $21.77 \mathrm{a}$ & $10.85 \mathrm{a}$ & $17.84 \mathrm{a}$ & $5.83 \mathrm{ab}$ \\
PE & $0.97 \mathrm{a}$ & $50.85 \mathrm{a}$ & $25.38 \mathrm{a}$ & $7.65 \mathrm{a}$ & $10.61 \mathrm{a}$ & $4.54 \mathrm{ab}$ \\
PC & $0.36 \mathrm{a}$ & $46.05 \mathrm{a}$ & $21.75 \mathrm{a}$ & $9.08 \mathrm{a}$ & $15.86 \mathrm{a}$ & $6.90 \mathrm{ab}$ \\
PI & $1.08 \mathrm{a}$ & $50.21 \mathrm{a}$ & $25.13 \mathrm{a}$ & $8.10 \mathrm{a}$ & $10.31 \mathrm{a}$ & $5.17 \mathrm{ab}$ \\
PIE & $2.83 \mathrm{a}$ & $38.38 \mathrm{a}$ & $27.29 \mathrm{a}$ & $10.07 \mathrm{a}$ & $16.59 \mathrm{a}$ & $4.87 \mathrm{ab}$ \\
PIC & $0.36 \mathrm{a}$ & $54.41 \mathrm{a}$ & $20.72 \mathrm{a}$ & $7.11 \mathrm{a}$ & $12.72 \mathrm{a}$ & $4.86 \mathrm{ab}$ \\
\hline
\end{tabular}

$\mathrm{TAB}=$ testigo; $\mathrm{E}=$ estiércol; $\mathrm{C}=$ composta; $\mathrm{P}=$ planta; $\mathrm{I}=$ inóculo. Medias con letra diferente son estadísticamente diferentes $(\mathrm{p} \leq 0.05)$
En la estabilidad de los agregados de tepetates se encontró que ésta disminuía, lo cual demuestra la formación de agregados. Los agregados son menos estables que los fragmentos. Los agregados más estables se encuentran entre $2-3$ y $>5 \mathrm{~mm}$ de diámetro, lo cual favorece el desarrollo de las plantas (Velázquez et al., 2001). También debe considerarse que el tiempo de experimentación fue solamente de un año.

\subsection{Propiedades químicas}

Los análisis químicos mostraron que el valor de $\mathrm{pH}$ varió de 7.3 a 8.1. El valor más bajo fue el del testigo absoluto 
(TAB) y el más alto el del tratamiento de planta + inóculo + composta (PIC). Con relación al testigo absoluto hubo un incremento en el potencial de hidrógeno. Se presentaron diferencias significativas $(\mathrm{P} \leq 0.05)$ sólo entre PIC y la mayor parte de los tratamientos (Tabla 4). Esto pudo deberse a la aplicación de la composta, la cual tenía pH de 7.7 y era rica en bases, así como a la actividad de la planta, que favorece el intemperismo del tepetate con la consecuente liberación de las bases al medio.

El porcentaje de carbono orgánico varió de $0.07 \%$ a $1.86 \%$, en TAB y PIE respectivamente, además se observó que hay diferencias significativas $(\mathrm{P} \leq 0.05)$ entre el resto de los tratamientos y el PIE (Tabla 4); la variación fue de extremadamente pobre a media. El estiércol, y la materia orgánica en general, son factores importantes en el aumento del porcentaje de carbono. Las fuentes de $\mathrm{C}$ influyeron en la composición y concentración de compuestos del suelo, así como en su efectividad para la agregación a través de la asociación con cationes y partículas del suelo (Bronick y Lal, 2005).

La capacidad de intercambio catiónico (CIC) varió de 23.88 a $30.88 \mathrm{Cmol}^{+} \mathrm{kg}^{-1}$, debido principalmente a que el tepetate está constituido por cantidades significativas de minerales amorfos (Flores et al., 1992; Acevedo et al., 2002), lo que justifica el valor alto de la capacidad de intercambio. Los resultados observados en la Tabla 4 presentan incremento y disminución reducida en dicho parámetro.

Por otra parte, en las bases intercambiables, los valores del $\mathrm{Ca}^{2+}$ variaron de 10.25 a $15.00 \mathrm{Cmol}^{+} \mathrm{kg}^{-1}$. Sin embargo, los valores de este elemento son suficientes para la nutrición de las especies vegetales (Vázquez, 1997). La cantidad de $\mathrm{Mg}^{2+}$ varió de 4.25 a $8.13 \mathrm{Cmol}^{+} \mathrm{kg}^{-1}$. Se presentó una tendencia general al incremento en la cantidad de $\mathrm{Mg}^{2+}$ con relación al testigo absoluto. El incremento de la cantidad de $\mathrm{Mg}^{2+}$ se atribuye a la alteración de minerales ferromagnesianos en el tepetate (Flores et al., 1992). Los valores de $\mathrm{Na}^{+}$variaron de 1.53 a $2.80 \mathrm{Cmol}^{+} \mathrm{kg}^{-1}$. El valor más bajo lo presentó el tratamiento de testigo + estiércol (TE) y el valor más alto el de planta + estiércol (PE). En la Tabla 4 se observa que hubo diferencias significativas $(\mathrm{p} \leq$ 0.05 ) entre los tratamientos PE, PI, PIE y los tratamientos TE y TC, incrementándose la cantidad de $\mathrm{Na}^{+}$en los primeros, pero sin diferencias entre ellos. La concentración de $\mathrm{Na}^{+}$aumentó debido al intemperismo de feldespatos sódicos en el tepetate (Flores et al., 1992) y al efecto de la planta en dicho intemperismo, así como a la presencia de este elemento en el estiércol. Los valores de $\mathrm{K}^{+}$variaron

Tabla 3. Porcentaje de estabilidad de los agregados ( $\mathrm{mm})$

\begin{tabular}{cccccccc}
\hline Tratamiento & $>5$ & $5-3$ & $3-2$ & $2-1$ & $1-0.5$ & $0.5-0.25$ & $<0.25$ \\
\hline TAB & $22.10 \mathrm{a}$ & $3.93 \mathrm{a}$ & $20.42 \mathrm{a}$ & $10.19 \mathrm{a}$ & $13.92 \mathrm{a}$ & $5.40 \mathrm{a}$ & $24.03 \mathrm{a}$ \\
TE & $44.66 \mathrm{a}$ & $4.47 \mathrm{a}$ & $16.82 \mathrm{a}$ & $7.10 \mathrm{a}$ & $8.71 \mathrm{a}$ & $2.79 \mathrm{a}$ & $15.44 \mathrm{a}$ \\
TC & $24.53 \mathrm{a}$ & $2.58 \mathrm{a}$ & $12.55 \mathrm{a}$ & $9.87 \mathrm{a}$ & $16.11 \mathrm{a}$ & $6.83 \mathrm{a}$ & $27.53 \mathrm{a}$ \\
$\mathrm{P}$ & $28.50 \mathrm{a}$ & $3.13 \mathrm{a}$ & $12.68 \mathrm{a}$ & $9.39 \mathrm{a}$ & $14.54 \mathrm{a}$ & $5.56 \mathrm{a}$ & $26.20 \mathrm{a}$ \\
PE & $38.24 \mathrm{a}$ & $3.78 \mathrm{a}$ & $17.49 \mathrm{a}$ & $10.63 \mathrm{a}$ & $11.45 \mathrm{a}$ & $3.24 \mathrm{a}$ & $15.15 \mathrm{a}$ \\
PC & $35.20 \mathrm{a}$ & $2.97 \mathrm{a}$ & $12.85 \mathrm{a}$ & $7.81 \mathrm{a}$ & $12.44 \mathrm{a}$ & $5.18 \mathrm{a}$ & $23.54 \mathrm{a}$ \\
PI & $43.68 \mathrm{a}$ & $4.46 \mathrm{a}$ & $13.71 \mathrm{a}$ & $7.05 \mathrm{a}$ & $9.03 \mathrm{a}$ & $3.36 \mathrm{a}$ & $18.71 \mathrm{a}$ \\
PIE & $25.16 \mathrm{a}$ & $3.34 \mathrm{a}$ & $21.17 \mathrm{a}$ & $10.97 \mathrm{a}$ & $16.14 \mathrm{a}$ & $5.48 \mathrm{a}$ & $17.48 \mathrm{a}$ \\
PIC & $45.64 \mathrm{a}$ & $4.99 \mathrm{a}$ & $14.88 \mathrm{a}$ & $7.14 \mathrm{a}$ & $8.75 \mathrm{a}$ & $3.75 \mathrm{a}$ & $14.84 \mathrm{a}$ \\
\hline
\end{tabular}

$\mathrm{TAB}=$ testigo; $\mathrm{E}=$ estiércol; $\mathrm{C}=$ composta; $\mathrm{P}=$ planta; $\mathrm{I}=$ inóculo

Medias con letra diferente son estadísticamente diferentes $(\mathrm{p} \leq 0.05)$

Tabla 4. Propiedades químicas del tepetate al final del experimento

\begin{tabular}{|c|c|c|c|c|c|c|c|}
\hline Tratamiento & $\mathrm{pH}$ & $\begin{array}{c}\text { Carbono } \\
\text { orgánico } \\
(\%)\end{array}$ & ------- & $\mathrm{Ca}^{2+}$ & $\begin{array}{c}\mathrm{Mg}^{2+} \\
\mathrm{Cmol}^{+} \mathrm{kg}^{-}\end{array}$ & $\mathrm{Na}^{+}$ & $\mathrm{K}^{+}$ \\
\hline TAB & $7.3 \mathrm{~b}$ & $0.07 \mathrm{~b}$ & $28.51 \mathrm{a}$ & $12.00 \mathrm{a}$ & $4.25 \mathrm{a}$ & $1.74 \mathrm{ab}$ & $1.49 \mathrm{a}$ \\
\hline $\mathrm{TE}$ & $7.4 \mathrm{~b}$ & $0.51 \mathrm{~b}$ & $25.25 \mathrm{a}$ & $10.25 \mathrm{a}$ & $4.38 \mathrm{a}$ & $1.53 \mathrm{~b}$ & $2.04 \mathrm{a}$ \\
\hline $\mathrm{TC}$ & $7.5 \mathrm{~b}$ & $0.32 \mathrm{~b}$ & $26.75 \mathrm{a}$ & $12.75 \mathrm{a}$ & $5.75 \mathrm{a}$ & $1.55 \mathrm{~b}$ & $1.94 \mathrm{a}$ \\
\hline $\mathrm{P}$ & $7.7 \mathrm{ab}$ & $0.14 \mathrm{~b}$ & $30.88 \mathrm{a}$ & $12.75 \mathrm{a}$ & $7.50 \mathrm{a}$ & $2.48 \mathrm{ab}$ & $1.65 \mathrm{a}$ \\
\hline $\mathrm{PE}$ & $7.4 \mathrm{~b}$ & $0.63 \mathrm{~b}$ & $29.88 \mathrm{a}$ & $13.38 \mathrm{a}$ & $6.38 \mathrm{a}$ & $2.80 \mathrm{a}$ & $2.40 \mathrm{a}$ \\
\hline $\mathrm{PC}$ & $7.8 \mathrm{ab}$ & $0.30 \mathrm{~b}$ & $30.75 \mathrm{a}$ & $14.63 \mathrm{a}$ & $6.50 \mathrm{a}$ & $2.60 \mathrm{ab}$ & $1.83 \mathrm{a}$ \\
\hline PI & $7.7 \mathrm{ab}$ & $0.17 \mathrm{~b}$ & $29.25 \mathrm{a}$ & $15.00 \mathrm{a}$ & $8.13 \mathrm{a}$ & $2.73 \mathrm{a}$ & $2.07 \mathrm{a}$ \\
\hline PIE & $7.5 \mathrm{~b}$ & $1.86 \mathrm{a}$ & $30.13 \mathrm{a}$ & $11.13 \mathrm{a}$ & $7.63 \mathrm{a}$ & $2.69 \mathrm{a}$ & $2.02 \mathrm{a}$ \\
\hline PIC & $8.1 \mathrm{a}$ & $0.58 \mathrm{~b}$ & $23.88 \mathrm{a}$ & $12.75 \mathrm{a}$ & $4.63 \mathrm{a}$ & $1.89 \mathrm{ab}$ & $1.48 \mathrm{a}$ \\
\hline
\end{tabular}

$\mathrm{TAB}=$ testigo; $\mathrm{E}=$ estiércol; $\mathrm{C}=$ composta; $\mathrm{P}=$ planta; $\mathrm{I}=$ inóculo.

Medias con letra diferente son estadísticamente diferentes $(\mathrm{p} \leq 0.05)$ 
de 1.48 a $2.40 \mathrm{Cmol}^{+} \mathrm{kg}^{-1}$, en los tratamientos PIC y PE respectivamente. En la Tabla 4 se observa un incremento en la cantidad de este catión. Los valores que se presentaron se pueden deber a la presencia de las enmiendas y los minerales potásicos del tepetate (Hidalgo et al., 1997).

De manera general, las bases intercambiables son elementos importantes para el desarrollo de la planta. La distribución y el contenido de los elementos intercambiables $\left(\mathrm{Ca}^{2+} \mathrm{Mg}^{2+}, \mathrm{Na}^{+} \mathrm{y} \mathrm{K}^{+}\right)$siguen un esquema relacionado con la presencia y el intemperismo de los feldespatos y de las micas, los cuales se encuentran en la composición de los tepetates y son liberados paulatinamente al medio (Fassbender y Bornemisza, 1987). Las plantas también favorecen el intemperismo, ya que al absorber los nutrimentos inducen una diferencia en la concentración de iones, lo cual provoca que los elementos que se encuentran en el material se difundan a la solución del suelo, permitiendo así que se liberen los iones que se encuentran en su composición (Nortcliff, 1992).

\subsection{Propiedades bioquímicas}

En la Tabla 5 se presentan únicamente las diferentes propiedades bioquímicas de los tratamientos que alcanzaron los niveles más altos. Asimismo, dichos contenidos están en relación al contenido total de carbono orgánico de cada tratamiento. Para estos análisis se seleccionó la repetición con mayor porcentaje de carbono de cada tratamiento y se realizó por duplicado, por lo que no se aplicó el análisis estadístico de estos parámetros y únicamente se hace un análisis descriptivo.

El contenido de carbono total es pobre (Fassbender y Bornemisza, 1987). Con un valor de $18.6 \mathrm{gC} / \mathrm{kg}$ de tepetate, obtuvo el valor más alto el tratamiento PIE; TE y PIC muestran altos grados de mineralización del carbono por lo que hay pocos residuos orgánicos.

Los porcentajes más altos de materia orgánica libre (MOL) los presentó TC. Dicha materia orgánica se encuentra en porcentajes muy bajos en todos los tratamientos y representa restos de plantas y enmiendas que no han sido degradados (Howard et al., 1998).

Con respecto a la fracción coloidal de los ácidos prefúlvicos y prehúmicos, el valor más alto es para el tratamiento PIE con 1.34 y $3.30 \mathrm{gC} / \mathrm{kg}$ de tepetate, respectivamente. Dichos valores son muy bajos y pueden deberse a la tasa baja de humificación de las enmiendas (Day y Kathleen, 2001); considerando además que el tiempo de observación, un año, es limitado (Pajares et al., 2010). El carbono de las huminas se encuentra en mayor proporción formando complejos arcilla-substancias prefúlvicas, ya que son las que predominan en los diferentes tratamientos (García, 2002).

La relación $\mathrm{AF} / \mathrm{AH}$ en el tratamiento $\mathrm{PIC}$ es mayor que 1, debido al predominio de los ácidos prefúlvicos poco evolucionados con estructura alifática, por lo que hay más mineralización que humificación. Por el contrario, en los tratamientos TC y PIE la relación es menor que 1, donde existe una ligera evolución de las moléculas prehúmicas (García, 2002).

El porcentaje de polisacáridos que se encuentran en el PIE y PIC son muy bajos, debido a que son fuentes de carbono para los microorganismos. Sin embargo para TE y TC, donde no se aplicó inóculo, tienen los valores más altos.

La aplicación de estiércol, inóculo y planta favorecieron el contenido total y una mayor mineralización de carbono, reflejándose en la disminución de la materia orgánica libre y aumento ligero de ácidos prehúmicos en el tepetate.

La presencia de estas substancias es importante en el tepetate, ya que pueden servir como indicadores de su calidad, dado que al inicio el tepetate presentó cantidades muy bajas de materia orgánica, por lo que no están presentes o se encuentran en cantidades mínimas y su seguimiento permitirá dar alternativas para mejorar su aprovechamiento (MacCarthy, 2001).

\subsection{Comportamiento de la especie vegetal}

En el rendimiento de la planta solamente en la altura se manifestaron diferencias significativas $(\mathrm{p} \leq 0.05)$. Así, el tratamiento planta + composta (PC) obtuvo la altura mayor y el de planta + estiércol (PE), la menor. Tanto en biomasa aérea como subterránea no hubo efecto de los tratamientos (Tabla 6).

\subsection{Micorrizas}

En relación a la colonización de las micorrizas, se observa en la Figura 3 que el menor porcentaje lo presentó el tratamiento de planta + inóculo + composta (PIC) con $19 \%$ y el mayor el menor el tratamiento planta + inóculo + estiércol (PIE) con $26.75 \%$. Esto puede deberse al mayor contenido de materia orgánica aportada por el estiércol,

Tabla 5. Propiedades bioquímicas determinadas ( $\mathrm{gC} / \mathrm{kg}$ suelo)

\begin{tabular}{cccccccc}
\hline Tratamiento & $\begin{array}{c}\text { Carbono } \\
\text { total }\end{array}$ & $\begin{array}{c}\text { Materia orgánica } \\
\text { libre }\end{array}$ & $\begin{array}{c}\text { Ácidos } \\
\text { prefúlvicos }\end{array}$ & $\begin{array}{c}\text { Ácidos } \\
\text { prehúmicos }\end{array}$ & Huminas & AF/AH & Polisacáridos* \\
\hline TE & 5.1 & 0.49 & 1.09 & 1.08 & 5.38 & 1 & 13.72 \\
TC & 3.2 & 1.12 & 0.29 & 0.77 & 4.28 & 0.37 & 15.62 \\
PIE & 18.6 & 0.33 & 1.34 & 3.3 & 5.88 & 0.4 & 4.83 \\
PIC & 5.8 & 0.84 & 0.63 & 0.18 & 2.19 & 3.5 & 3.44 \\
\hline
\end{tabular}

* \% de polisacáridos con respecto al Carbono total 
Tabla 6. Rendimiento de la planta

\begin{tabular}{cccc}
\hline Tratamiento & $\begin{array}{c}\text { Altura } \\
(\mathrm{cm})\end{array}$ & $\begin{array}{c}\text { Biomasa aérea } \\
(\mathrm{g})\end{array}$ & $\begin{array}{c}\text { Biomasa } \\
\text { subterránea } \\
(\mathrm{g})\end{array}$ \\
\hline $\mathrm{P}$ & $103.25 \pm 6.9 \mathrm{ab} *$ & $13.09 \pm 0.95 \mathrm{a}$ & $8.34 \pm 0.95 \mathrm{a}$ \\
$\mathrm{PE}$ & $74.50 \pm 16.11 \mathrm{~b}$ & $16.28 \pm 4.38 \mathrm{a}$ & $8.71 \pm 3.67 \mathrm{a}$ \\
PC & $114.50 \pm 16.76 \mathrm{a}$ & $13.83 \pm 3.70 \mathrm{a}$ & $11.45 \pm 3.08 \mathrm{a}$ \\
PI & $88.75 \pm 16.44 \mathrm{ab}$ & $10.30 \pm 3.72 \mathrm{a}$ & $7.97 \pm 4.50 \mathrm{a}$ \\
PIE & $104.00 \pm 7.79 \mathrm{ab}$ & $17.73 \pm 2.43 \mathrm{a}$ & $8.07 \pm 2.57 \mathrm{a}$ \\
PIC & $93.50 \pm 13.63 \mathrm{ab}$ & $12.97 \pm 4.30 \mathrm{a}$ & $7.95 \pm 4.30 \mathrm{a}$ \\
\hline
\end{tabular}

*Valores con diferente letra son estadísticamente diferentes $(\mathrm{p} \leq 0.05)$ Los valores son medias de $n=4$

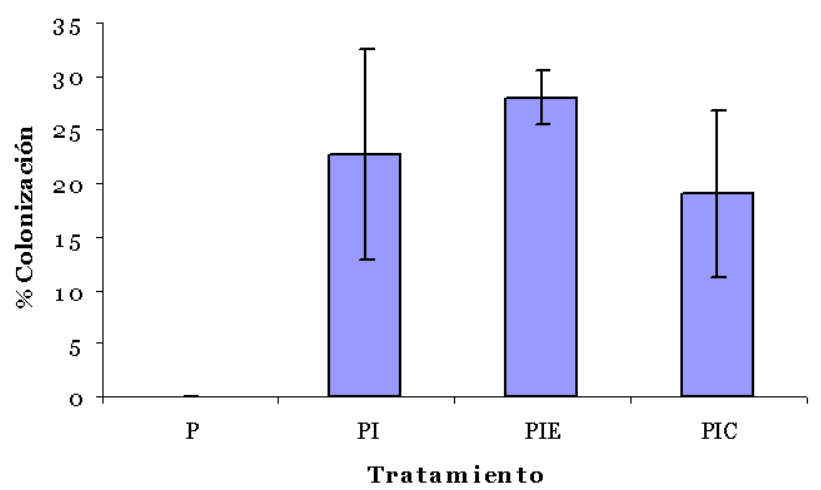

Figura 3. Colonización de las micorrizas. Promedio $(n=4)$

equivalente a $72 \mathrm{Mg} \mathrm{ha}^{-1}$, contra $40 \mathrm{Mg} \mathrm{ha}^{-1}$ de la composta. Se ha observado que la colonización micorrízica total aumenta de manera notoria por efecto de la aplicación de materia orgánica (Ferrera et al., 1997).

\section{Conclusiones}

Finalmente se concluye que: 1) Los tratamientos modifican la distribución original de agregados, especialmente existe una disgregación (fragmentos y agregados órgano-minerales) en la fracción $2-5 \mathrm{~mm}$ y su consecuente agregación en la fracción $0.25-1 \mathrm{~mm}$. 2) La presencia de fracciones $>10 \mathrm{~mm}$ se debe a la disgregación y posterior agregación del material. 3) $\mathrm{El} \mathrm{pH}$ ligeramente alcalino se mantuvo dentro del valor apropiado para el desarrollo vegetal; el tratamiento con planta, inóculo y estiércol (PIE) obtuvo el contenido más alto de carbono orgánico; los valores altos y homogéneos de la capacidad de intercambio catiónico (CIC) fueron debidos fundamentalmente a los minerales amorfos del tepetate y secundariamente, a la materia orgánica; en las bases intercambiables, solamente en el $\mathrm{Na}^{+}$hubo incremento por efecto de tratamientos. 4) La humificación es incipiente por el predominio de estructuras alifáticas y poca condensación, debido al poco tiempo para que evolucionen las moléculas; los contenidos de polisacáridos para los tratamientos TE y TC son los más altos, pero no fueron suficientes para aumentar el número y tamaño de agregados. 5) En el rendimiento de la planta, el durazno manifestó una respuesta muy homogénea en las variables estudiadas. 6) El mayor porcentaje de colonización micorrízica se obtuvo en el tratamiento con mayor contenido de materia orgánica (PIE).

\section{Agradecimientos}

A la DGAPA (PAPIIT) UNAM, por el apoyo financiero de este proyecto. Código IN219205-2.

\section{Referencias}

Acevedo, O.A., Flores, D., 2000, Genesis of white fragipans of volcanic origin: Revista Mexicana de Ciencias Geológicas, 17, 152-162.

Acevedo, O.A., Cruz, M., Cruz, E., 2002, Distribución de óxidos de Fe, $\mathrm{Al}$ y Si en horizontes endurecidos de origen volcánico: Agrociencia, 36, 401-409.

Acevedo, O.A., Velázquez, A., Flores, D., 2001, Agregación por especies vegetales y abonos orgánicos en tepetates fracturados en condiciones de invernadero: Terra Latinoamericana, 19, 363-373.

Báez-Pérez, A., Etchevers-Barra, J.D., Prat, C., Hidalgo-Moreno, C., 2007, Formation of aggregates and carbon sequestration in ameliorated tepetates in the Rio Texcoco basin, México: Revista Mexicana de Ciencias Geológicas, 24, 487-497.

Bronick, C.J., Lal, R., 2005, Soil structure and management: a review: Geoderma, 124, 3-22.

Day, M., Kathleen, S., 2001, Biological, chemical and physical processes of composting, en Stofella, P.J., Kahn, B.A. (eds.), Compost utilization in horticultural cropping systems: Boca Raton, Florida, E.U.A., Lewis Publishers, 17-50.

Denef, K., Six, J., Bossuyt, H., Frey, S.D., Elliott, E.T., Merckx, R., Paustian, K., 2001, Influence of wet dry cycles on the interrelationship between aggregate, particulate organic matter, and microbial community dynamics: Soil Biology \& Biochemistry, 33, 1599-1611.

Fassbender, H.W., Bornemisza, E., 1987, Química de suelos con énfasis en suelos de América Latina: San José, Costa Rica, Instituto Interamericano de Cooperación para la Agricultura, $420 \mathrm{p}$.

Ferrera, R., González C., M.C., Rodríguez, M., 1993, Manual de Agrobiología:Texcoco, México, Colegio de Posgraduados, 142 p.

Ferrera, R., Ortiz, A., Delgadillo, J., Santamaría S., 1997, Uso de la materia orgánica en la recuperación de tepetates y su influencia en los microorganismos, en Zebrowski C., Quantin, P., Trujillo, G. (eds.), III simposio internacional sobre suelos volcánicos endurecidos, 1996: Quito, Ecuador, 225-237.

Flores, D., González, A., Alcalá, J.R., Gama, J.E., 1991, Los tepetates: Revista de Geografía INEGI, 3, 37-42.

Flores, D., Alcalá, J.R., González, A., Gama, J.E., 1992, Suelos con fragipán de origen volcánico en clima semicálido y subhúmedo - El caso del Noreste del estado de Morelos, México: Revista del Instituto de Geología - UNAM, 10, 151-163.

García, A., Flores, D., García, N.E., Ferrera, R., 2009, Efecto de enmiendas orgánicas, higuera y micorriza sobre las características de un tepetate: Terra Latinoamericana, 26, 309-315.

García, C.N.E., 2002, Importancia de la materia orgánica en la conservación y degradación del suelo, en $2^{\circ}$ Simposio Internacional sobre Degradación del Suelo-XXXI Congreso Nacional de la Ciencia del Suelo: Torreón, Coahuila, México, Sociedad Mexicana de Ciencia del Suelo, 59-66.

Hidalgo, C., Quantin, P., Elsass, F., 1997, Caracterización mineralógica de los tepetates tipo fragipán del Valle de México, en Zebrowski C., Quantin, P., Trujillo, G. (eds.), III simposio internacional sobre suelos volcánicos endurecidos, 1996: Quito, Ecuador, 65-72. 
Howard, P.J.A., Howard, D.M., Lowe, L.E., 1998, Effects of tree species and physico-chemical conditions on the nature of soil organic matter: Soil Biology and Biochemistry, 30, 285-297.

IUSS working group WRB (IUSS-WRB), 2006, World reference base for soil resources 2006: Roma, Italia, World Soil Resources Reports No. 103, FAO, 145 p.

Ivarson, K.C., Sowden, F.J., 1962, Methods for the analysis of carbohydrate material in soil, I. Colorimetric determination of uronic acids, hexoses and pentoses: Soil Sciences, 94, 245-250.

Jackson, M.L. 1970, Análisis Químico de Suelos: Barcelona, España, Omega, $662 \mathrm{p}$.

JMP, 2002, JMP ${ }^{\circledR}$ Statistics and Graphics Guide Version 5: Cary, Carolina del Norte., E.U.A., SAS Institute, 315-334.

Kemper, W.D., Rosenau, R. C., 1986, Aggregate Stability and Size Distribution, en Black, C.A. (ed.), Methods of Soil Analysis Part 1 Physical and mineralogical methods: Madison, Wisconsin, E.U.A., American Society of Agronomy and Soil Science Society of America, 425-442.

Kononova, M.N., 1982, Materia orgánica del suelo: su naturaleza, propiedades y métodos de investigación: Barcelona, España, OIKOS-TAU, $365 \mathrm{p}$.

MacCarthy, P., 2001, The principles of humic substances: Soil Science, $166,738-751$.

Nelson, D.W., Sommers, L.E., 1996, Total carbon, organic carbon and organic matter, en Sparks, D.L., Page, A.L., Helmke, P.A., Loeppert, R.H., Soluanpour, P.N., Tabatabai, M.A., Johnston, C.T., Summer, M.E. (eds.), Methods of soil analysis Part 3 Chemical methods: Madison, Wisconsin, E.U.A., American Society of Agronomy and Soil Science Society of America, 961-1010.
Nortcliff, S., 1992, Formación del suelo y características de los perfiles edáficos, en Wild, A. (ed.), Condiciones del suelo y desarrollo de las plantas según Russel: Madrid, España, Mundi-Prensa, 177-221.

Pajares, S., Gallardo, J.F., Marinari, S., Etchevers, J.D., 2010, Indicadores bioquímicos de calidad en tepetates cultivados del Eje Neovolcánico Mexicano: Agrociencia, 44, 121-134.

Shiel, R.S., Adey, M.A., Lodder, M., 1988, The effect of successive wet/ dry cycles on aggregate size distribution in clay texture soil: Journal of Soil Science, 39, 71-80.

Vázquez A., 1997, Guía para interpretar el análisis químico del agua y suelo: Chapingo, Estado de México, México, Universidad Autónoma Chapingo - Departamento de suelos, $31 \mathrm{p}$.

Velázquez, A.S., Flores, D., Acevedo, O.A., 2001, Formación de agregados en tepetate por influencia de especies vegetales: Agrociencia, 35, 311-320.

Zebrowski, C., 1992, Los suelos volcánicos endurecidos en América Latina: Terra, número especial 10, 15-23.

Manuscrito recibido: Agosto 17, 2010.

Manuscrito corregido recibido: Junio 16, 2011.

Manuscrito aceptado: Junio 18, 2011. 